\title{
EL DERECHO INFORMÁTICO EN EL PROYECTO DE ACUERDO TRIPS DE LA RUEDA URUGUAY
}

Carlos M. Correa

\section{El proyecto de Acuerdo TRIPs}

En diciembre de 1991, el secretario general del GATT sometió a los países miembros de esa organización una ambiciosa propuesta global de acuerdo multilateral para regular diversas facetas del comercio internacional. Tal propuesta incluyó - por primera vez en la historia del GATT - normas relativas a propiedad intelectual, contenidas en un proyecto de acuerdo sobre «Trade related aspects of intellectual property rights» (TRIPs).

El proyecto de Acuerdo TRIPs, en caso de ser finalmente aprobado, influirá de manera sustancial en múltiples aspectos de la propiedad intelectual, por tres razones principales.

En primer lugar, dicho proyecto contiene normas que serán aplicables de manera uniforme en más de cien países miembros del GATT. Desde que ellas están formuladas como standards mínimos, su instrumentación asegurará un cierto «piso» en la protección de los derechos intelectuales en casi todo el mundo.

Segundo, tal Acuerdo será el más comprensivo entre los convenios internacionales sobre la materia, pues incluye disposiciones sobre derechos de autor (y derechos vecinos), diseños industriales, marcas, indicaciones de origen, patentes, diseño de circuitos integrados e información confidencial. Los únicos títulos no abordados — sino de modo marginal - han sido los derechos de obtentor y los modelos de utilidad.

Tercero, la adopción de TRIPs permitirá a cualquier país miembro del 
GATT acudir al sistema de resolución de controversias del GATT y, en su caso, aplicar represalias comerciales en cualquier rubro de bienes o servicios si se determina la inobservancia de alguno de los standards mínimos de protección.

El proyecto de Acuerdo no establece distinción entre las normas aplicables de países desarrollados y en desarrollo, excepto en lo que respecta a la fecha de entrada en vigor de las nuevas disposiciones (normas transitorias).

En materia de derecho informático, el Acuerdo TRIPs contiene disposiciones que afectan tres temas clave que se tratan sucesivamente más abajo, en relación con la protección de: a) los programas de computación; b) los bancos de datos, y c) los circuitos integrados.

\section{La protección del software}

\section{a) La situación actual}

La definición de standards internacionales de protección mínima para el software ha sido, sin duda, uno de los objetivos importantes de los países que han impulsado la negociación de un Acuerdo sobre TRIPs. Si bien en la última década numerosos países desarrollados y en desarrollo han adoptado el derecho de autor como forma principal de protección (Correa, 1990a), diversos problemas no han encontrado una solución plena, particularmente en la perspectiva de la industria de software con una vasta acción internacional.

Primero, no todos los países han resuelto definitivamente la cuestion del régimen legal aplicable al software. En algunos, la cuestión sigue irresuelta (p. ej. Tailandia), en tanto en otros (varios países de América Latina), la jurisprudencia aún es frágil y no ha habido cambios legislativos que introduzcan de manera explícita tal protección ${ }^{1}$.

1 Sólo Brasil, México y Chile cuentan con normas legales recientes que clarifican la aplicación del derecho de autor a los programas de computación. Se han dictado también decretos en tal sentido en Colombia y Uruguay (Correa, 1991a). 
Segundo, aun en países en donde la aplicación del derecho de autor ha sido admitida en principio, existen dificultades de «enforcement» debido a la naturaleza de los procesos judiciales o a la escasa significación de las penas. La «piratería», más controlada hoy que hace diez años, no ha dejado sin embargo de constituir un fenómeno extendido, incluso en países industrializados.

Tercero, diferencias en los regímenes nacionales de derecho de autor condujeron a resultados prácticos disímiles. El ejemplo más notable, tal vez, es el de la interpretación restrictiva del concepto de «originalidad» en el derecho alemán (y suizo), con la consecuente desprotección de una gran parte del software protegible en otros países bajo un concepto más elástico (basado en la existencia de un esfuerzo intelectual).

Cuarto, la dicotomía idea/expresión ha resultado de compleja aplicación en relación con una obra funcional como el software y la extensión de la protección suscita diferencias entre países. Así, en tanto en Japón y los Estados Unidos la ingeniería inversa tendería a ser aceptada (Pilny, 1992), en los países miembros de la CEE — sobre la base de la reciente Directiva sobre la Protección de los Programas de Computación ${ }^{2}$ - ella sólo es admisible para la inetopreabilidad de un programa creado de manera independiente (artículo 6).

Quinto, las diferencias de interpretación sobre el alcance de los derechos se han manifestado incluso en el sistema judicial de los Estados Unidos. La citada decisión en Whelan vs. Jaslow (1986, U. S. Court Of Appeals, Third Circuit), que admitió la protección de la estructura y organización de un programa, ha sido recientemente revertida en Computer Associates vs. Altai (junio de 1992, U. S. Court of Appeals, Second Circuit).

Sexto, y por último, si bien se admite en general la protección del programa-objeto como un «trade secret», una forma adicional de protección puede basarse en el sistema de patentes. Al menos en los Estados Unidos, la solicitud de patentes respecto de programas de computación ha comenzado a constituir una tendencia visible, la que se acentuará quizás en la medida

2 Directiva del Consejo 91/250/CEE del 14.5.91. 
en que persista la incertidumbre en relación con el alcance de la protección vía copyright (Business Week, 1992, p. 49).

\section{b) Las reglas de TRIPs}

\section{i) Protección como obra literaria}

El proyecto de Acuerdo TRIPs (artículo 9.1) obliga a la observancia de los artículos 1 a 21 y del Apéndice de la Convención de Berna, en su versión de 1971. Si bien tal convención no hace referencia expresa a los programas de computación, ha prevalecido en general el criterio de que ellos están amparados por sus disposiciones ${ }^{3}$. Para evitar toda duda, el artículo 10 de dicho proyecto de Acuerdo establece que los «computer programs, whether in source or object code, shall be protected as literary works under the Berne Convention (1971)».

La norma citada no sólo representa un punto final a cualquier especulación sobre la aplicación del Convenio de Berna al software. Ella zanja también la cuestión —-debatida en la doctrina y jurisprudencia— de la categoría de obra intelectual a la que pertenecen o se equiparan los programas de computación ${ }^{4}$. Ellos deben ser protegidos «como» obras literarias, lo que implica descartar soluciones que, como la de la ley francesa de 1986, se basan en la equiparación de hecho del software a obras de arte aplicadas, con sujeción a un plazo menor de protección (veinticinco años).

\section{ii) Alcance de la protección}

En la propuesta del Japón al grupo negociador de TRIPs —en línea con la legislación de aquel país-, el artículo 10.1 referido se completaba con la aclaración de que «such protection shall not extend to ideas, procedures,

3 En tal sentido, el «Protocolo» a la Convención de Berna, bajo consideración en el ámbito de la OMPI, no haría sino hacer explícita dicha cobertura (ver OMPI, 1992).

${ }^{4}$ La controversia al respecto se centró principalmente en la posibilidad de reconocer carácter de obra literaria a un programa no legible por el hombre, por ejemplo, incorporado en una memoria ROM. 
methods of operation or mathematical concepts». El propósito de este agregado era trazar una línea demarcatoria más clara entre lo que debe entenderse como apropiable y lo que no debe caer bajo el derecho de propiedad, demarcación que tiene particular importancia a la hora de juzgar la legitimidad de la ingeniería inversa y la posibilidad de imitar la estructura interna y organización de un programa.

Si bien la propuesta referida no encontró consenso, un texto similar al indicado se incorporó en el artículo 9.2 del proyecto de Acuerdo, mas con un alcance que se extiende a todos los derechos amparados bajo la Convención de Berna y no sólo a los programas de computación. Dicho artículo establece que: «Copyright protection shall extend to expressions and not to ideas, procedures, methods of operation or mathematical concepts as such». La expresión «as such» de este artículo in fine puede introducir un sesgo restrictivo en la interpretación de la norma, que no estaba presente en la propuesta japonesa.

Otra norma relevante para juzgar la extensión de los derechos, pero también de alcance general, está contenida en el artículo 13, de conformidad con el cual, «PARTIES shall confine limitations or exceptions to exclusive rights to certain special cases which do not conflict with a normal exploitation of the work and do not unreasonably prejudice the legitimate interests of the right holder».

La admisibilidad o no de la descompilación e ingeniería inversa de los programas de computación, así como la extensión de la protección más allá de la expresión (incluyendo a la estructura interna), dependerá de la interpretación que las legislaciones nacionales brinden a las normas indicadas. En todo caso, es claro que el proyecto de Acuerdo ha eludido resolver de manera precisa esos aspectos, con lo cual su poder armonizador en esa materia será limitado.

\section{iii) Derechos de alquiler}

El artículo 11 del proyecto comentado introduce, por primera vez en un tratado multilateral ${ }^{5}$, la noción de «rental rights» (derechos de alquiler) como

s El único antecedente importante de esta disposición se encuentra en la ya 
uno de los elementos del haz de derechos exclusivos del titular de un programa de computación. Según dicho artículo, «In respect of at least computer programs and cinematographic works, a Party shall provide authors and their successors in title the right to authorise or prohibit the commercial rental to the public of originals or copies of their copyright works... In respect of computer programs, this obligation does not apply to rentals where the program itself is not the essential object of the rental».

El reconocimiento de los derechos de alquiler no admite excepciones -a diferencia de lo que el proyecto de Acuerdo prevé para las obras cinematográficas-, si bien se aclara su inaplicabilidad en el caso de que el software sea parte no esencial de otro producto alquilado (p. ej. un automóvil). El reconocimiento expreso de tales derechos parece tener particular importancia en los regímenes basados en el concepto de «copyright», mas no para aquellas legislaciones -como las inspiradas en el derecho continental- que típicamente otorgan al autor un derecho exclusivo de distribución.

\section{iv) Duración}

En materia de duración de la protección, el Acuerdo TRIPs puede tener un importante efecto armonizador de las legislaciones nacionales con relación a las obras que no pertenecen a personas físicas. En el caso del software, lo más frecuente es que los programas comercializados (particularmente los de tipo «standard») sean desarrollados por empresas u otras entidades, a quienes la ley atribuye -directa o indirectamente- la titularidad de aquéllos.

Según el artículo 12 del proyecto de Acuerdo, las obras -excepto las fotográficas y de arte aplicado- tendrán una tutela no inferior a cincuenta años contados desde el fin del año de su publicación y, en ausencia de ésta, de cincuenta años contados desde el fin del año de su creación.

\section{v) Otros aspectos}

Como se señaló más arriba, el patentamiento del software puede convertirse

mencionada Directiva de la CEE de mayo de 1991 (artículo 4 c). En el plano nacional, se destaca la «Computer Software Rental Amendments Act» de 1990, de los Estados Unidos. 
en una alternativa o complemento a la protección vía derecho de autor. El Acuerdo de TRIPs - a diferencia de la Convención europea de patentes y de diversas legislaciones- no excluye la posibilidad de que el software sea patentado. De acuerdo con el artículo 27.1, «patents shall be available for any inventions, whether products or procesess, in all fields of technology...».

Por otro parte, tal Acuerdo daría nacimiento al primer cuerpo internacional de normas sobre «undisclosed information», una forma de protección de particular importancia para los programas de computación comercializados exclusivamente en su modalidad de programa-objeto. La sección 7 contempla la aplicación de la disciplina de la competencia desleal, sobre la base de definiciones y reglas inspiradas en la legislación estadounidense sobre «trade secrets».

Diversas disposiciones de la Parte III del Acuerdo —que no se analizan aquí- sobre el ejercicio y «enforcement» de los derechos de propiedad intelectual, pueden ser de relevancia para la protección del software en su faz práctica. Así, por ejemplo, el artículo 51 faculta a detener en las aduanas «pirated copyright goods», en tanto el artículo 61 obliga a los países miembros del GATT a disponer de procedimientos y sanciones penales para la «piratería de copyright en escala comercial».

\section{Compilaciones de datos}

El artículo 10.2 del proyecto de Acuerdo dispone:

"Compilations of data or other material, whether in machine readable or other form, which by reason of the selection or arrangement of their contents constitute intellectual creations shall be protected as such. Such protection, which shall be without prejudice to any copyright subsisting in the data or material itself».

La emergente industria de bancos de datos y la creciente importancia económica de éstos, han generado demandas por una protección más explícita y efectiva de aquéllos, tanto en lo que respecta a la reproducción y distribución de la información compilada como a la extracción o re-utilización no autorizada del contenido de un banco de datos. 
Si bien algunas legislaciones, como la de los Estados Unidos (artículo 103 de la U.S. Copyright Act) y del Japón (Ley 64 de 1986) ${ }^{6}$, prevén la protección de las compilaciones de datos en cuanto tales, la Convención de Berna sólo se refiere a la de «colecciones de obras literarias o artísticas tales como enciclopedias y antologías». En al ámbito de la CEE se ha elaborado una propuesta de Directiva para la «Protección Legal de las Bases de Datos» con base en el derecho de autor ${ }^{7}$, mas limitada a aquéllas operadas por medios electrónicos y a los derechos económicos de los titulares. Además del típico derecho a impedir la reproducción no autorizada, dicha propuesta prevé un «derecho especial» para prevenir la extracción o re-uso no autorizado, del total o una parte sustancial, de la información almacenada.

Según se desprende del citado artículo 10.2 del proyecto de Acuerdo, las negociaciones en TRIPs concluyeron con un texto que, por un lado, suplementa la Convención de Berna en tanto alude a compilaciones «de datos» y no sólo a la de obras autorales, y, por el otro, no se limita a las bases de datos computarizadas, sino que abarca también las manuales. A diferencia de la propuesta de Directiva de la CEE, además, la elaborada en TRIPs no incluye referencia alguna a «derechos especiales» para evitar la extracción o re-utilización de datos.

Con el alcance expuesto, el proyecto de Acuerdo claramente inscribe la protección de las compilaciones de datos en el ámbito del copyright y en el marco de los derechos exclusivos que típicamente se reconoce bajo aquel régimen. La cobertura de las compilaciones, sea que ellas fueren computarizadas o manuales, justifica, por cierto, que no se fuere más lejos en cuanto a los derechos reconocidos, sin perjuicio de la posibilidad, para cualquier país, de conceder una protección adicional (como la propuesta en la CEE).

\section{Circuitos integrados}

La protección del diseño (o «layout») de los circuitos integrados, está dando

6 Con relación a la legislación japonesa, ver Karjala (1986).

7 El «Creen Paper» sobre propiedad intelectual de la Comisión de las Comunidades Europeas había sugerido, en 1988, la posibilidad de desarrollar un régimen sui generis de protección de las bases de datos (ver Clark, 1991). 
lugar a una situación sin precedentes en el dominio de la propiedad intelectual. Tras cerca de cinco años de negociaciones, la Conferencia Diplomática de Washington adoptó, en mayo de 1989, un Tratado para la protección de dichos diseños - vía una modalidad sui generis - por una abrumadora mayoría de los países participantes. Estados Unidos y Japón, principales productores mundiales de semiconductores e interesados en el establecimiento de tal protección, no suscribieron el texto del tratado acordado ${ }^{8}$.

El proyecto de Acuerdo TRIPs, como se ha visto, suplementa la Convención de Berna en diversos aspectos (también lo hace con las Convenciones de Roma y París). En el caso de los circuitos integrados, y aun cuando el Convenio de Washington no está en vigor, dicho proyecto obliga a los países miembros a observar sus disposiciones con las salvedades y agregados que — no sorprendentemente- reflejan las objeciones de los Estados Unidos y el Japón a dicho tratado.

En primer lugar, el referido proyecto de Acuerdo incluye una norma que fue explícitamente rechazada en la Conferencia de Washington por los países en desarrollo, en virtud de la cual el ámbito de la protección no sólo se extiende a los diseños y a los circuitos integrados que los incorporan, sino a los artículos que incorporan tales circuitos, «en la medida que continúe conteniendo un diseño ilegalmente reproducido» (artículo 36).

Segundo, se prevé la excepción del «infractor inocente». No se considerará infractor a quien adquiriere un circuito integrado o un artículo que incorpore un diseño reproducido ilegalmente sin conocer ni tener razonables fundamentos para conocer de tal reproducción ilegítima. El artículo 37.1 del proyecto de Acuerdo agrega, sin embargo, que «after the time that such person has received sufficient notice that the layout-design was unlawfully reproduced, he may perform any of the acts with respect to the stock on hand or ordered before such time, but shall be liable to pay to the holder of the right a sum equivalent to a reasonable royalty such as would be payable under a freely negotiated license in respect 6 of such a layout-design».

8 Sobre estas negociaciones y las implicaciones del Tratado de Washington, ver Correa (1190 b) y Kitagawa (1992). 
El pago de una regalía por el infractor inocente fue, en la Conferencia Diplomática de Washington, uno de los puntos más objetados, debido a la imposibilidad que tienen adquirentes de los más diversos productos industriales, particularmente en los países en desarrollo, de verificar la legitimidad de los diseños incorporados en los «chips». La solución adoptada por el proyecto de Acuerdo puede constituirse, en la práctica, en una barrera al comercio y en una amenaza permanente sobre los compradores de bienes electrónicos y otros que incorporan componentes informáticos. Lo lógico y justo sería, en todo caso, que la acción del titular del diseño se dirija contra el fabricante del producto que lo incorpora y no contra quien lo adquiere (salvo que lo hiciera de mala fe).

Tercero, el artículo 37.1 hace aplicables a las licencias obligatorias de diseños de circuitos integrados (uno de los puntos de discordia para los Estados Unidos en el Tratado de Washington), las detalladas disposiciones que el proyecto de Acuerdo contiene sobre «otros usos» (no autorizados por el titular) de las patentes de invención. Ello implica, entre otros aspectos, la necesidad de una negociación previa con el titular y la evaluación de caso por caso de las solicitudes de licencia.

Por último, en tanto el tratado aludido estableció una duración mínima de los derechos de ocho años, el proyecto de Acuerdo extiende tal plazo a diez años.

\section{Principales conclusiones}

El análisis efectuado permite extraer las siguientes conclusiones principales.

La aprobación del Acuerdo TRIPs significará un paso importante en la universalización del ya predominante patrón legislativo basado en la aplicación del derecho de autor para la protección del software y en la consideración de éste como obra literaria. Las normas alcanzadas amplían y refuerzan los derechos para combatir las copia ilegítima («piratería») de programas de computación en los países en que el marco normativo es aún frágil o impreciso. En cambio, el Acuerdo no aportará soluciones y criterios uniformes para los aspectos tan cruciales, como controvertidos, relacionados con la extensión de la protección y la admisibilidad de la extensión de la protec- 
ción y la admisibilidad de la ingeniería inversa. No es difícil presumir que estos vacíos son el resultado de la complejidad de los temas a ser abordados, y de las significativas divergencias que, según se observó más arriba, separan incluso a la jurisprudencia en los Estados Unidos.

En materia de compilaciones de datos, el proyecto de Acuerdo ofrece la posibilidad de universalizar su proteccion vía el derecho autoral -con lo que se excluye la vía de un régimen sui generis- . Si bien deja igualmente sin resolver aspectos vinculados con actos diferentes a la reproducción, tal como la extracción y re-utilización de los datos, la solución parece la más adecuada dada la amplia cobertura de la norma propuesta (bases de datos computarizadas y manuales) y la dificultad existente para trazar la línea divisoria entre lo que es protegible y lo que no lo es.

En lo que respecta a diseños de circuitos integrados, las modificaciones y complementos introducidos al Tratado de Washington reflejan, por un lado, la pérdida de capacidad negociadora que, en corto término, sufrieron los países en desarrollo ( y los que coincidieron con ellos en la Conferencia de Washington), al pasar el tema del ámbito de la OMPI al del GATT. Por el otro, tales cambios no representan una mejora de las normas multilaterales sobre la materia y pueden, particularmente, afectar indebidamente a los adquirentes de bienes que incorporan «chips».

Como conclusión general, el proyecto de Acuerdo TRIPs influirá ciertamente en la configuración de componentes importantes del derecho informático en un gran número de países. En el área del software, la mayor influencia se dará en los países en desarrollo que aún no hubieren definido claramente su protección vía derecho de autor. En los otros dos temas tratados, la influencia será aún más abarcativa, pues el proyecto introduce soluciones que son nuevas o que significan una definición más firme a la existente en la actualidad tanto en países industrializados como en desarrollo.

Sin perjuicio de ello, no puede verse al proyecto de Acuerdo comentado como el punto final a los debates y diferencias que aún prevalecen en relación con múltiples temas de la propiedad intelectual en el área de la informática. Si bien tal proyecto define la modalidad y ciertos contenidos de la protección, son múltiples los aspectos que separarán aún a las empresas - según sus posiciones relativas en el mercado y su capacidad tecnológi- 
ca-, a los expertos y a los países con distintos grados de desarrollo y percepciones sobre el modo de favorecer la producción y el comercio de bienes informáticos. 


\section{Bibliografía}

\section{BUSINESS WEEK}

1992 «Bit by bit, software protection is eroding», julio 20 , pp. 49-50.

CLARK, C.

1991 «The value of information - The protection of compilations, including databases», Managing Intellectual Property, noviembre, pp. 10-12.

CORREA, C.

1990 Tecnología y desarrollo de la informática en el contexto Norte-Sur, Eudeba, Buenos Aires, 220 pp.

CORREA, C.

1991 a «Evolución reciente del derecho informático en América Latina», La Ley, tomo A, Buenos Aires, pp. 848.

CORREA, C.

$1991 \mathrm{~b}$ «Intellectual property in the field of integrated circuits: implications for developing countries», World Competition, Geneva, pp. 83-102.

KARJALA, D.

1986 «Protection of computer databases under japanese law», European Intellectual Property Review, vol. 8, $\mathrm{N}^{\circ} 9$, setiembre, pp. 267.

KITAGAWA, Z.

1991 «Treaty on intellectual property in respect of integrated circuits - A failed success-», in Restschrift für Werner Lorenz, Mohr, Tübingen, pp. 646-675. 
PILNY, K.

1992 «Legal aspects of interfaces and reverse engineering Protection in Germany, the United States and Japan», IICD, Munich, pp. 196-218.

OMPI (Organización Mundial de la Propiedad Intelectual)

1992 «Cuestiones relativas a un eventual protocolo del Convenio de Berna», Parte I, Ginebra, julio, pp. 1-25. 